Wilfrid Laurier University

Scholars Commons @ Laurier

Physics and Computer Science Faculty

Publications

Physics and Computer Science

3-10-2015

\title{
Signatures of Chaos in the Dynamics of Quantum Discord
}

\author{
Vaibhav Madhok \\ Wilfrid Laurier University \\ Vibhu Gupta \\ University of Waterloo \\ Denis-Alexandre Trottier \\ University of Waterloo \\ Shohini Ghose \\ Wilfrid Laurier University, sghose@wlu.ca
}

Follow this and additional works at: https://scholars.wlu.ca/phys_faculty

Part of the Physics Commons

\section{Recommended Citation}

V. Madhok, V. Gupta, D.-A. Trottier, and S. Ghose, "Signatures of chaos in the dynamics of quantum discord", Physical Review E 91, 032906 (2015).

This Article is brought to you for free and open access by the Physics and Computer Science at Scholars Commons @ Laurier. It has been accepted for inclusion in Physics and Computer Science Faculty Publications by an authorized administrator of Scholars Commons @ Laurier. For more information, please contact scholarscommons@wlu.ca. 


\title{
Signatures of chaos in the dynamics of quantum discord
}

\author{
Vaibhav Madhok, ${ }^{1, *}$ Vibhu Gupta,,${ }^{2,3}$ Denis-Alexandre Trottier, ${ }^{3}$ and Shohini Ghose ${ }^{1,3, \dagger}$ \\ ${ }^{1}$ Department of Physics and Computer Science, Wilfrid Laurier University, Waterloo, Ontario N2L 3C5, Canada \\ ${ }^{2}$ Department of Physics and Astronomy, University of Waterloo, Ontario N2L 3G1, Canada \\ ${ }^{3}$ Institute for Quantum Computing, University of Waterloo, Ontario N2L 3G1, Canada
}

(Received 7 August 2014; revised manuscript received 3 January 2015; published 10 March 2015)

\begin{abstract}
We identify signatures of chaos in the dynamics of discord in a multiqubit system collectively modelled as a quantum kicked top. The evolution of discord between any two qubits is quasiperiodic in regular regions, while in chaotic regions the quasiperiodicity is lost. As the initial wave function is varied from the regular regions to the chaotic sea, a contour plot of the time-averaged discord remarkably reproduces the structures of the classical stroboscopic map. We also find surprisingly opposite behavior of two-qubit discord versus entanglement of the two qubits as measured by the concurrence. Our results provide evidence of signatures of chaos in dynamically generated discord.
\end{abstract}

DOI: 10.1103/PhysRevE.91.032906

PACS number(s): 05.45.Mt, 03.67.Ac, 03.65.Ud

\section{INTRODUCTION}

Classical chaos has been widely studied in a variety of contexts, including weather patterns, population dynamics, and chemical reactions [1]. Chaos in classical systems is characterized by sensitivity to initial conditions, which means that nearby trajectories separate exponentially fast with a rate given by the Lyapunov exponents of the system. The above characterization of chaos fails in quantum mechanics as the overlap of two state vectors undergoing unitary evolution due to Schrödinger's equation is constant with time. A natural question that arises is in regard to how to characterize chaos at the quantum level. This has led to the development of the field of quantum chaos: the study of how chaos manifests itself in the quantum regime. Signatures of chaos in quantum systems have been explored in the context of level statistics of chaotic Hamiltonians [2,3], the dynamics of open quantum systems undergoing measurement or decoherence [4,5], and hypersensitivity of a system to perturbations [6,7]. In the past decade, there has been considerable interest in the role of dynamical chaos in entanglement generation. This is central to the understanding of the emergence of the classical world from the underlying quantum mechanics and the role of entanglement in the irreversibility of dynamical evolution [8]. Moreover, such questions are important as entanglement is a crucial resource for quantum information processing [9-12].

However, entanglement does not completely capture the quantum correlations of a system, and neither does it seem to be the only reason behind the quantum advantage in quantum information processing. Quantum discord aims to fill this gap and capture essentially all the quantum correlations in a quantum state using information-theoretic measures [1315]. There is a considerable interest in quantum discord as recent studies show that it may account for the speedup in the performance of certain quantum algorithms compared to classical ones [16].

In this work, we provide the first evidence of signatures of chaos in the the dynamical behavior of discord in a quantum

\footnotetext{
*vmadhok@gmail.com

†sghose@wlu.ca
}

kicked top. An advantage of the quantum kicked top is that for a given angular momentum $j$, it can be regarded as a quantum simulation of a collection of $N=2 j$ spin-half particles whose evolution is restricted to the symmetric subspace under particle exchange. Thus, we have a multiqubit system where the collective behavior of the qubits is governed by the kicked-top Hamiltonian. Another advantage of this approach is that it allows us to study discord between any two qubits and compare it to pairwise entanglement between two qubits or to bipartite entanglement between two qubits and the remaining qubits. Here we present results showing various signatures of chaos in the dynamics of discord between any two qubits. The discord dynamics exhibits regular, quasiperiodic behavior in a regular regime but not in a chaotic regime. A contour plot of the time-averaged discord reproduces the classical phase space structures. We find a surprising relationship between two-qubit discord and two-qubit concurrence-a measure of pairwise entanglement. When discord increases, concurrence decreases and vice versa. The two-qubit discord is robust and remains nonzero in a chaotic regime, whereas the concurrence quickly decreases to zero. The quantum kicked top was experimentally realized recently [17], and our simulations are performed using parameters that are experimentally accessible using current technology. In the light of recent advances in quantum simulations and computation using superconducting qubits [18], we believe that our findings can be realized to explore quantum signatures of classical chaos even for systems that are deep in the quantum regime.

\section{BACKGROUND}

\section{A. Quantum kicked top}

The quantum kicked top is described by the Hamiltonian $[2,17,19]$

$$
H=\frac{\kappa}{2 j \tau} J_{z}^{2}+p J_{y} \sum_{n=-\infty}^{n=+\infty} \delta(t-n \tau) .
$$

Here $J_{x}, J_{y}$, and $J_{z}$ are components of the angular-momentum operator $\mathbf{J}$. The time between periodic kicks is given by $\tau$. Each kick is a rotation about the $y$ axis by an angle $p . \kappa$ is the strength of a twist applied between kicks and is also the 
chaoticity parameter: as $\kappa$ is increased, the degree of chaoticity increases. Since the kick is in the form of delta kicks, we can express the Floquet map (evolution from kick to kick) as a sequence of operations given by

$$
U_{\tau}=\exp \left(-i \frac{\kappa}{2 j \tau} J_{z}^{2}\right) \exp \left(-i p J_{y}\right) .
$$

For a given value of angular momentum $j$, the Hilbert space dimension is $2 j+1$. The finite dimension of the Hilbert space makes it possible to explore the dynamics without the need for truncation of the space.

The classical limit of this map can be obtained by writing the Heisenberg equations of motion for the expectation values of $J_{x}, J_{y}$, and $J_{z}$ and factorizing higher moments of the angularmomentum operators in the limit of large $j$. The resulting equations describe the motion of an angular-momentum vector on the surface of a sphere. The dynamics can be understood as a rotation by a fixed angle $p$ about the $y$ axis by angle $p$, followed by a rotation about the $z$ axis by an angle proportional to the $z$ component of the angular momentum. This sequence of transformations can result in chaotic dynamics due to the lack of enough constants of motion. In our analysis, we fix $p=\pi / 2$ and choose $\kappa$ to be our chaoticity parameter. As we vary $\kappa$ from 0 to 6 , the classical limit of the dynamics change from highly regular to completely chaotic. In the quantum description, as the dynamics becomes globally chaotic, and for $j \gg 1$, the Hamiltonian can be modelled as a random matrix selected from the appropriate ensemble [2]. It is this randomness that leads to the analog of ergodic mixing for quantum systems.

We can think of the total angular momentum $j$ as the sum of the angular momenta of $N=2 j$ individual spin-half particles or qubits. The qubits are identical and the system remains unchanged under the exchange of any two qubits. Hence the state vector is restricted to a symmetric subspace spanned by the basis states $\{|j, m\rangle ;(m=-j,-j+1, \ldots, j)\}$ with $j=$ $N / 2$.

In order to explore the quantum dynamics and compare it to the classical limit, we must pick an initial condition for the dynamics. In the classical case, the initial condition is a set of coordinates $\theta$ and $\phi$ which specify the initial direction of the classical angular-momentum vector. The uncertainty principle does not allow us to pick a corresponding quantum initial condition. Instead, we construct a minimum uncertainty state vector such that the expectation values of $J_{x}, J_{y}$, and $J_{z}$ define a vector pointing along the direction $\theta, \phi$. Such states are the spin-coherent states, which can be expressed as [19-22]

$$
|\theta, \phi\rangle=R(\theta, \phi)|j, j\rangle ; \quad-\pi \leqslant \phi \leqslant \pi, 0 \leqslant \theta \leqslant \pi,
$$

where

$$
R(\theta, \phi)=\exp \left\{i \theta\left[J_{x} \sin \phi-J_{y} \cos \phi\right]\right\}
$$

with the expectation value of $\mathbf{J}$ given by

$$
\langle\theta, \phi|\mathbf{J} / j| \theta, \phi\rangle=(\sin \theta \cos \phi, \sin \theta \sin \phi, \cos \theta) .
$$

And the relative variance of $\mathbf{J}$ in a state $|\theta, \phi\rangle$ is [19]

$$
\left(1 / j^{2}\right)\left\{\left\langle\theta, \phi\left|\mathbf{J}^{2}\right| \theta, \phi\right\rangle-\langle\theta, \phi|\mathbf{J}| \theta, \phi\rangle^{2}\right\}=1 / j .
$$

This is the minimum uncertainty possible from the angularmomentum commutation relations and approaches zero as $j$ becomes very large.

\section{MEASURES OF QUANTUM CORRELATIONS}

\section{A. Entanglement}

For a particular value of $j$, the system can be decomposed into $N=2 j$ qubits. To quantify correlations among these qubits, we trace out $N-2$ qubits from the rest of the system [23]. The two-qubit state, $\rho$, thus obtained is a mixed state and the von Neumann entropy of this two-qubit state, defined as $E_{V}=-\operatorname{Tr}(\rho \ln \rho)$, captures how the two qubits are entangled with the rest of the qubits [24]. Pairwise entanglement between the two qubits can be quantified by concurrence [25]. Concurrence is the entanglement of formation for a two-qubit state. If we have a bipartite system, $\rho, \in \mathcal{H}^{A B}$, comprising systems A and $\mathrm{B}$, then entanglement of formation of the composite mixed state is defined as the minimum average entanglement of an ensemble of pure states that represents $\rho$ [25].

$$
E_{f}(\rho)=\min \left(\sum_{i} p_{i} E\left|\psi_{i}\right\rangle\left\langle\psi_{i}\right|\right),
$$

where $\left|\psi_{i}\right\rangle$ are pure states and $E\left(\left|\psi_{i}\right\rangle\left\langle\psi_{i}\right|\right)$ represents the entanglement as quantified by the von Nuemann entropy of one of the subsystems. For the two-qubit case, it has a simplified expression defined as

$$
C=\max (0, \Lambda)
$$

where $\Lambda=\lambda_{1}-\lambda_{2}-\lambda_{3}-\lambda_{4}$, and $\lambda_{i}$ are the eigenvalues in decreasing order of the matrix $\rho\left(\sigma_{2} \otimes \sigma_{2}\right) \rho^{*}\left(\sigma_{2} \otimes \sigma_{2}\right)$. $\sigma_{2}$ is a Pauli matrix and $\rho^{*}$ is the complex conjugate of $\rho$.

\section{B. Quantum discord}

Quantum discord is a measure that captures all quantum correlations, including and beyond entanglement in a quantum state [13]. The approach to do this is to remove the classical correlations from the total correlations in a system. A measure of total correlations in a bipartite quantum system $\rho_{A B}$ is the quantum mutual information,

$$
\mathcal{I}(A: B)=\mathcal{H}(A)+\mathcal{H}(B)-\mathcal{H}(A, B),
$$

where $\mathcal{H}(\cdot)$ is the von Neumann entropy, $\mathcal{H}(\rho) \equiv$ $-\operatorname{Tr}(\rho \log \rho)$. A definition of mutual information for classical probability distributions based on Bayes's rule is

$$
I(A: B)=H(A)-H(A \mid B) .
$$

Here the conditional entropy $H(A \mid B)$ is the average of the Shannon entropies of $A$, conditioned on the values of $B$, and reflects the ignorance in $A$ given the state of $B$. In the quantum case, we can describe measurements on $B$ by a POVM (positive-operator valued measure) set $\left\{\Pi_{i}\right\}$, such that the conditioned state of $A$ given outcome $i$ is

$$
\rho_{A \mid i}=\operatorname{Tr}_{B}\left(\Pi_{i} \rho_{A B}\right) / p_{i}, \quad p_{i}=\operatorname{Tr}_{A, B}\left(\Pi_{i} \rho_{A B}\right) .
$$

The POVMs are a set of Hermitian, positive, and complete operators and represent the most general quantum measurements on a quantum system [26]. The corresponding entropy is then $\tilde{\mathcal{H}}_{\left\{\Pi_{i}\right\}}(A \mid B) \equiv \sum_{i} p_{i} \mathcal{H}\left(\rho_{A \mid i}\right)$, from which one can write the quantum mutual information as $\mathcal{J}_{\left\{\Pi_{i}\right\}}(A$ : $B)=\mathcal{H}(A)-\tilde{\mathcal{H}}_{\left\{\Pi_{i}\right\}}(A \mid B)$. Maximizing this over all $\left\{\Pi_{i}\right\}$, we obtain $\mathcal{J}(A: B)=\max _{\left\{\Pi_{i}\right\}}\left(\mathcal{H}(A)-\tilde{\mathcal{H}}_{\left\{\Pi_{i}\right\}}(A \mid B)\right) \equiv \mathcal{H}(A)-$ 
$\tilde{\mathcal{H}}(A \mid B)$, where $\tilde{\mathcal{H}}(A \mid B)=\min _{\left\{\Pi_{i}\right\}} \tilde{\mathcal{H}}_{\left\{\Pi_{i}\right\}}(A \mid B)$. The minimum is achieved using rank 1 POVMs since the conditional entropy is concave over the set of convex POVMs [27]. Hence we arrive at a definition for quantum discord:

$$
\begin{aligned}
\mathcal{D}(A: B) & =\mathcal{I}(A: B)-\mathcal{J}(A: B) \\
& =\mathcal{H}(B)-\mathcal{H}(A, B)+\min _{\left\{\Pi_{i}\right\}} \tilde{\mathcal{H}}_{\left\{\Pi_{i}\right\}}(A \mid B),
\end{aligned}
$$

with $\left\{\Pi_{i}\right\}$ being rank 1 POVMs. Quantum discord is non-negative for all quantum states [13,27], and it is subadditive [28].

\section{DYNAMICS OF QUANTUM CORRELATIONS}

In order to study the connection between discord and chaos in the kicked top, we use the multiqubit representation of the system as discussed above. We trace out two qubits whose state and discord are calculated after every application of the Floquet map. Since all the qubits are identical, this represents the discord between any two qubits of the system. We choose as initial states the minimum uncertainity spin-coherent states, which can be characterized by angle $\theta$ and $\phi$. We take for the initial conditions different points in the classical phase space for $p=\pi / 2$ and $\kappa=3$ (Fig. 1). We choose to take points corresponding to $(\theta, \phi)=$ $(2.25,0.63),(2.25,0.90),(2.25,1.05)$, and $(\theta, \phi)=(2.25,2.00)$. As can be seen from the classical phase space, these four points correspond to a fixed point, a point chosen in the regular island, an "edge of chaos" point or point on the boundary of the regular island and the chaotic sea [29], and a point chosen in the middle of the chaotic sea. In Fig. 2 we plot the discord dynamics for different values of $\phi$ for $\theta=2.25$. It can be clearly noticed that as we move towards the chaotic region, the long-term periodic modulation is lost. Also, it clearly shows that chaotic initial conditions lead to a higher value of average dynamically generated discord. The dynamics of discord for these specific four coordinates are shown separately in Fig. 3. For a coherent state initialized at a fixed point and in a regular island, discord

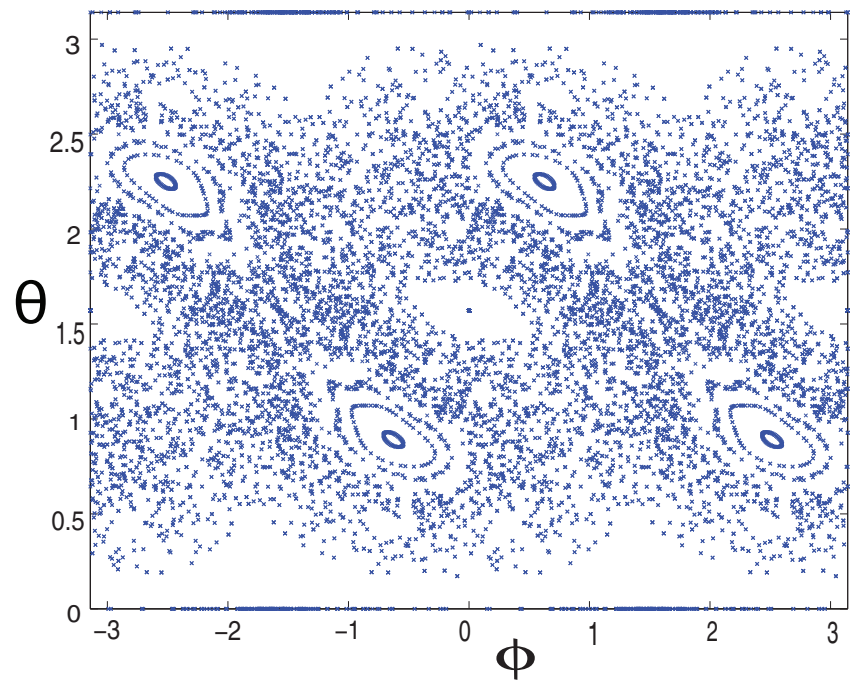

FIG. 1. (Color online) Classical stroboscopic map for the kicked top. The direction of the angular-momentum vector is plotted after each kick for different initial conditions with $p=\pi / 2, \kappa=3$.

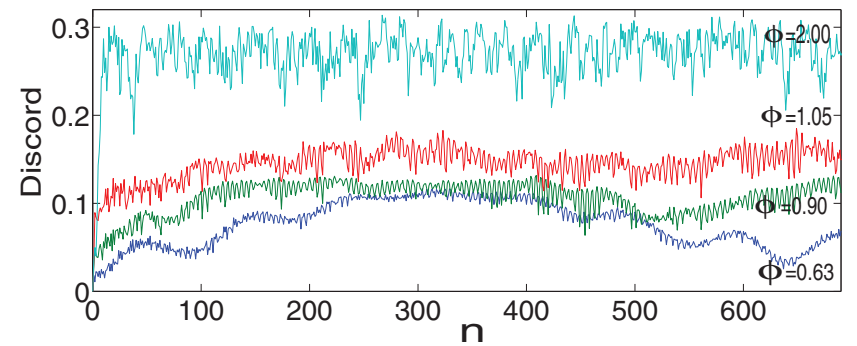

FIG. 2. (Color online) Dynamical evolution of discord for initial SCS with $\theta=2.25$ and different $\phi$. Here we have taken parameters as $\kappa=3$ and $j=40$.

increases at a slow rate and exhibits quasiperiodic oscillatory behavior. For a spin-coherent state initially in the chaotic sea $(\theta=2.25, \phi=0.63)$, the discord increases more rapidly and reaches a quasisteady state. The periodic modulation of discord dynamics is lost as the initial conditions are scanned from the regular region to the chaotic sea in the classical phase space. This demonstrates that there is a correlation between discord dynamics and regular versus chaotic regions of the classical phase space where the quantum state is initialized. It is remarkable that we see these signatures even though we operate with very few qubits $(j=4$ which corresponds to just eight qubits). Such a quantum regime is achievable in current experiments [17]. As we increase the value of $j$, the system approaches the classical limit and the signatures of chaos become clearer.

In order to further understand the signatures of chaos in the evolution of discord, we look at the time-averaged value of discord as we scan through different initial conditions. A contour plot of the time-averaged discord as a function of the initial spin-coherent state. Figure 4 clearly reproduces the
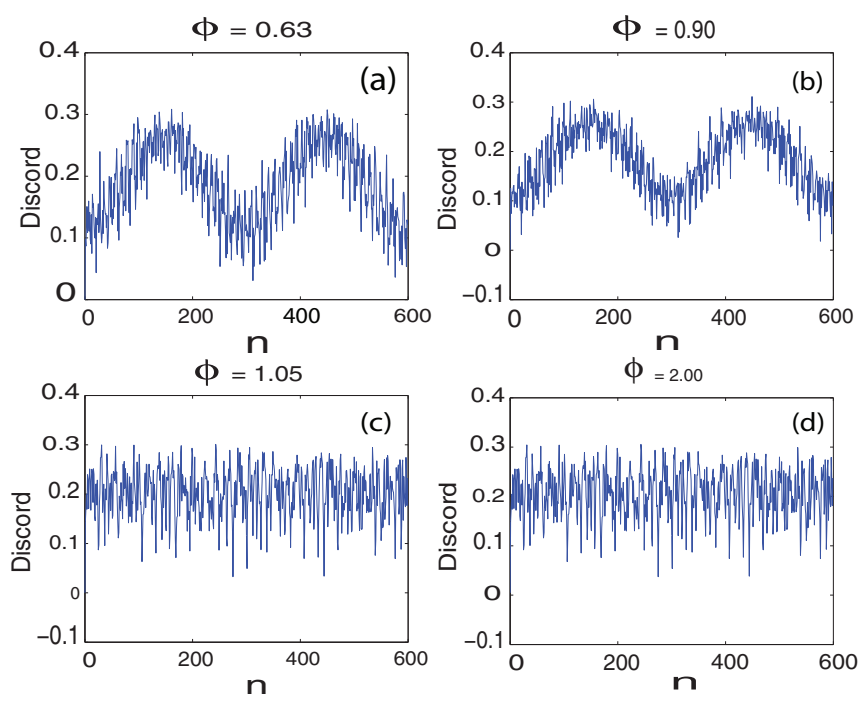

FIG. 3. (Color online) Dynamically generated discord as a function of the number of applications of the Floquet map for $j=4$ and $\kappa=3$. (a) Fixed-point initial condition $(\theta=2.25, \phi=0.63)$. (b) Regular initial condition $(\theta=2.25, \phi=0.90)$. (c) Edge-of-chaos initial conditions $(\theta=2.25, \phi=1.05)$. (d) Chaotic initial condition $(\theta=2.25, \phi=2.00)$. 

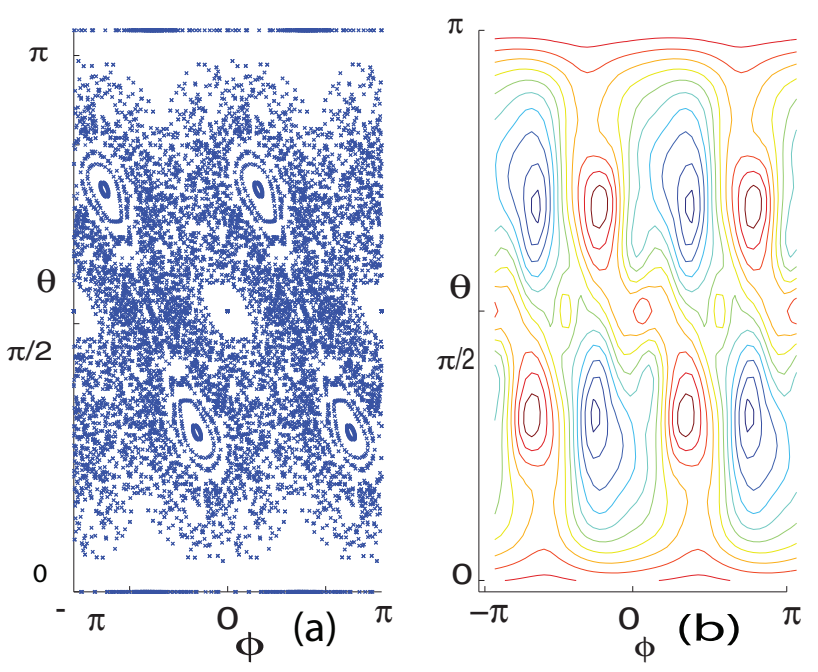

FIG. 4. (Color online) Side-by-side comparison showing dynamically generated discord as a remarkable signature of classical chaos in a mixed phase space $(p=\pi / 2, \kappa=3$, and $j=40)$. (a) Classical phase space, Poincaré section. (b) Long-time average discord, $D$, as a function of mean coordinate of the initial projected coherent state. A weighted average of $D$ according to the measure on phase space gives the value of $\bar{D}=0.275$ in the chaotic sea and $\bar{D}=0.143$ in the regular islands.

regular and chaotic structure of the classical phase space and shows remarkably strong correlation between structures in the classical mixed phase space and the time-averaged discord plot. Chaotic initial conditions generally go to a higher average value than regular initial conditions, with the smallest values of discord generation near the classical fixed points. Additionally, all initial conditions in the chaotic sea saturate to nearly the same average discord.

Long-time average discord for states localized in the chaotic sea is higher than those localized deep inside regular islands. As one gradually approaches the border between a regular island and the chaotic sea, the dynamically generated discord for an initial condition inside a regular island becomes comparable to that for an initial state located inside the chaotic sea [Figs. 3(c) and 3(d)]. Thus, discord as a signature of chaos effectively differentiates between the features of the classical phase space and regular islands from the chaotic sea, while leaving the border between the regular island and the chaotic sea murky. Therefore, as discussed above and as Figs. 3(c) and 3(d) show, one needs to be careful with the initial states at the border [30] that has an average value of discord comparable to the value inside the chaotic sea. This is especially true for lower values of $j$ such that the system is far from the classical limit. While classical chaos leads to infinitely intricate structures in the phase space, in quantum mechanics, the Plank's constant, $\hbar$, limits the scale for such structures. Our resolution of the phase space is determined by the Plank's constant. Therefore, quantum discord is a universal signature of chaos if one considers it as a tool to demarcate regular islands from the chaotic sea in a coarse-grained fashion.

In order to compare dynamically generated discord as a function of chaos, we consider the same initial state while increasing the chaos in the system gradually. Figure 5 shows

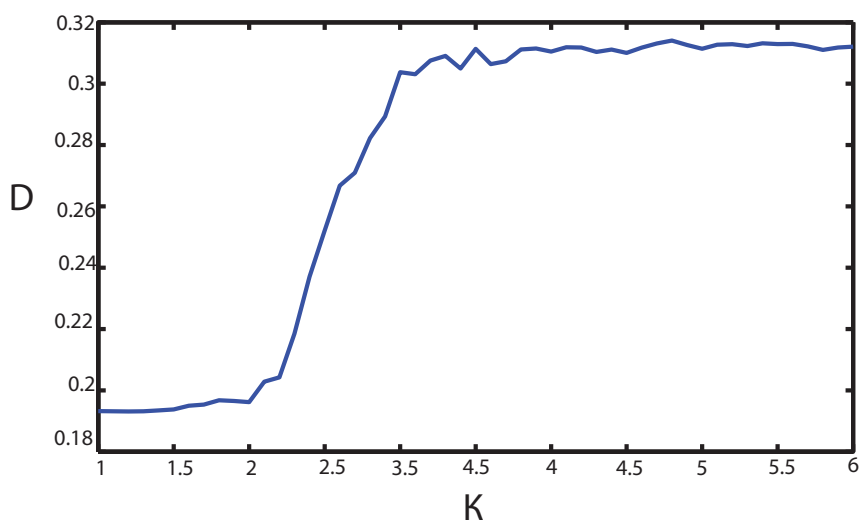

FIG. 5. (Color online) Average discord, $D$, as a function of the chaoticity parameter, $\kappa$, for the kicked top for $j=100$. The average is calculated over the first 350 kicks. The initial state is the same as in Fig. 3(c), given by $(\theta=2.25, \phi=1.05)$.

the average discord generated for the same initial state as in Fig. 3(c), given by $(\theta=2.25, \phi=1.05)$, as we gradually increase the chaoticity parameter. We see a strong correlation between the degree of chaos in the system and the average value of discord generated. Therefore, for a fixed family of maps and sufficiently large spin size, the generation of discord is a function of chaos in the system.

For the mixed phase space $(p=\pi / 2, \kappa=3)$, the value of long-time discord is almost the same for all initial states in the chaotic sea. To find the average discord of the chaotic and regular regions, we take a grid of coherent states across the phase space. Each point on the grid is classified as as "regular" or "chaotic" by the Lyapunov exponent of the classical dynamics. Weighting these values according to the measure on phase space gives us an average discord of $\bar{D}=0.275$ in the chaotic sea and $\bar{D}=0.143$ in the regular islands. Therefore, using the average value of discord, one can distinguish regular islands from the chaotic sea.

Our studies are similar to signatures of chaos observed previously in time-averaged entanglement [11]. We also note that the average value of discord for initial spin-coherent states in different parts of the chaotic sea reaches roughly the same value. To confirm this we took a slice of the graph and plotted long-time average discord for constant $\theta=2.25$ and varying $\phi$ (Fig. 6). We see that the fixed-point region has a significantly lower value of discord compared to the chaotic region. There is gradual change as we cross from the regular islands into the chaotic sea. The time-averaged discord thus can be used to identify the edge of chaos [12].

To understand the nature of measures of correlations such as discord and entanglement and their relationship to each other, we next compare the discord dynamics with the entanglement dynamics. The two-qubit discord quantifies the correlation of these two qubits among themselves, while the two-qubit von Neumann entropy quantifies the entanglement of these two qubits with the rest of the system. Figure 7 shows discord dynamics compared to the von Neumann entropy and concurrence dynamics. We find that discord dynamics mirror the entropy dynamics very well and behaves opposite to that of concurrence dynamics. When concurrence is high, discord 

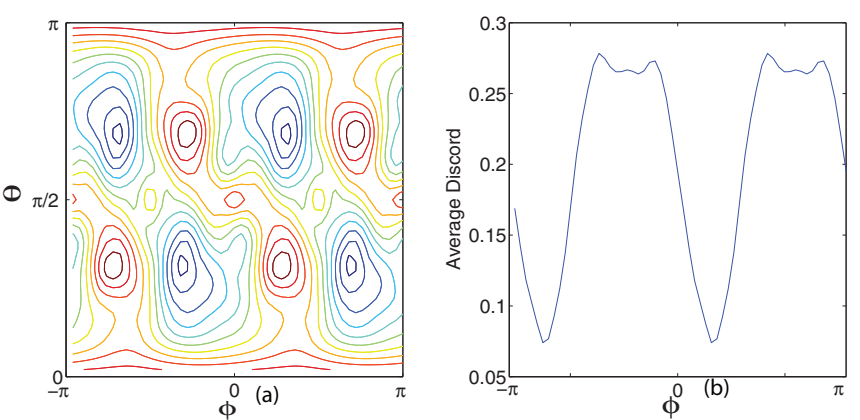

FIG. 6. (Color online) (a) Contour plot of discord for $j=40$. Blue regions indicate regions of low average discord. (b) Average discord for $\theta=2.25$ and varying $\phi$. Comparison to classical phase space shows that chaotic region initial conditions lead to higher average discord.

is low and vice versa. Our results show that although both concurrence and discord are measures of quantum correlations, they are two separate quantities. We feel that this is an important finding and, to the best of our knowledge, has not been reported elsewhere. Our results suggest a similarity between various measures of correlations in a quantum system and demand a detailed and systematic study.

\section{A. Chaos and random matrix theory}

Quantum chaos is intimately connected with the theory of random matrices [2]. As one approaches large Hilbert space dimensions, and the corresponding classical limit of the quantum system exhibits global chaos, the eigenstates and eigenvalues of the quantum Hamiltonian operator have the statistical properties of appropriately chosen random matrices. The appropriate ensemble depends on the symmetries of the system, for example, whether the quantum system has a time-reversal symmetry [2]. The kicked top has a time-reversal symmetry. The time-reversal operator, $T$, for the kicked top can be given the standard form

$$
T=U K,
$$

where $U=\exp \left(i p J_{y}\right) \exp \left(i \pi J_{x}\right)$ and $K$ is the complex conjugation with respect to a standard representation. Since $T^{2}=1$, there is no Kramer's degeneracy [2,3]. The kicked top is time-reversal invariant [2] has the consequence that the Floquet operator has the statistical properties of a random matrix
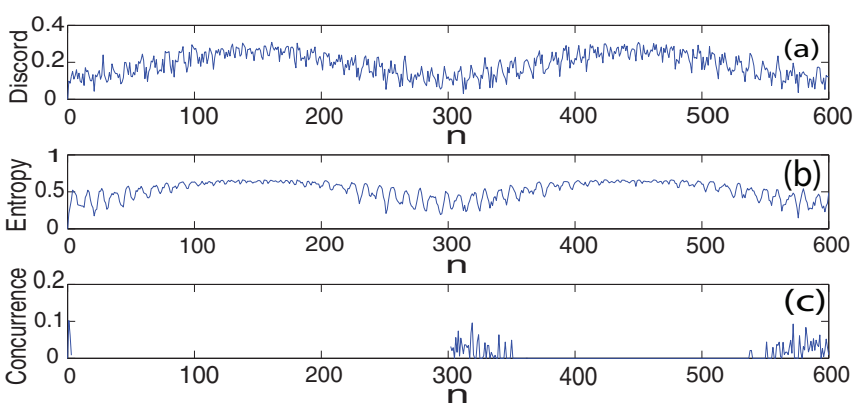

FIG. 7. (Color online) (a) Discord dynamics. (b) Entropy dynamics and (c) concurrence dynamics for $j=4$.
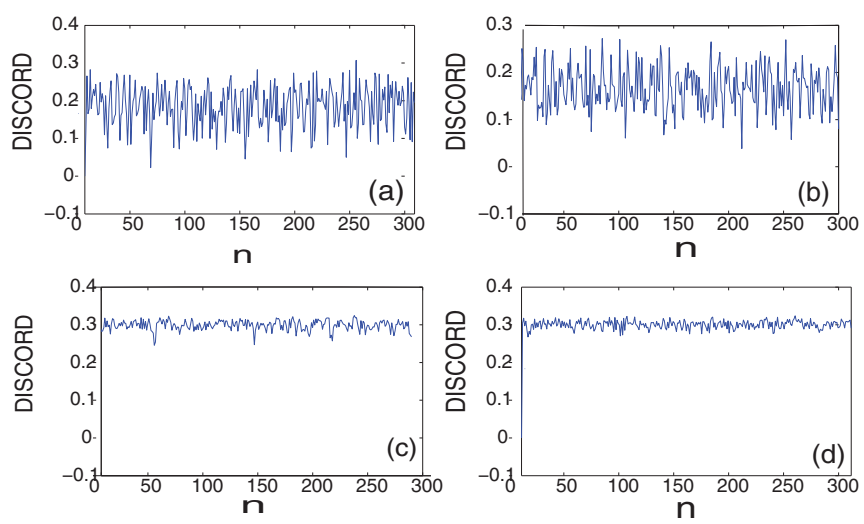

FIG. 8. (Color online) Dynamically generated discord as a function of the number of applications of (a) the Floquet map corresponding to global chaos for $j=4$. (b) A random unitary chosen from the COE for $j=4$. (c) The Floquet map corresponding to global chaos for $j=40$. (d) A random unitary chosen from the COE for $j=40$.

chosen from the circular orthogonal ensemble (COE). The eigenvectors of these random operators have well-defined statistical properties [31]. Since the COE ensembles are invariant under a group of orthogonal transformations, the eigenvectors must be uniformly distributed on a vector space according to the Haar measure that is invariant under that group. However, the case of the dynamically evolved state differs [11]. In addition to the time-reversal symmetry, the kicked top has a parity symmetry, $R=\exp \left\{-i \pi j_{y}\right\}$, that commutes with the Floquet map. In the basis of the parity operator, the Floquet map has a block-diagonal structure having two blocks associated with the positive- or negative-parity eigenvalues. Due to the parity symmetry, the kicked top is statistically equivalent to a block-diagonal random matrix (block diagonal in the basis in which the parity operator is diagonal) whose blocks (corresponding to positive and negative eigenvalues) are sampled from the COE [32].

We use a block-diagonal COE as the appropriate ensemble of random matrices for the kicked-top Hamiltonian to evolve the system and compare the dynamics of discord with that of when the system is acted upon by the kicked-top Hamiltonian. Figure 8(b) shows the evolution of 2-qubit discord for a system of 8 qubits when acted upon by a random unitary, while Fig. 8(d) shows discord evolution for 80 qubits. We find that the discord dynamics under the action of random unitary is very similar to the discord dynamics under the action of a kicked-top Hamiltonian in the chaotic regime [Figs. 8(a) and 8 (c), respectively]. Interestingly, the fluctuations around the mean value of quantum discord, as measured by the standard deviation, decline at the rate proportional to $1 / \sqrt{N}$, where $N$ is the number of qubits (Fig. 9). This is consistent with entropy calculations on random states and properties of random vectors in Hilbert space [33]. As the value of discord becomes sharply defined upon increasing the dimension of the chaotic system and, as a result, one can talk about the discord of a typical state.

This shows that the kicked top with parameters in the chaotic region effectively simulates a random unitary. This can have potential practical application as random unitary have 


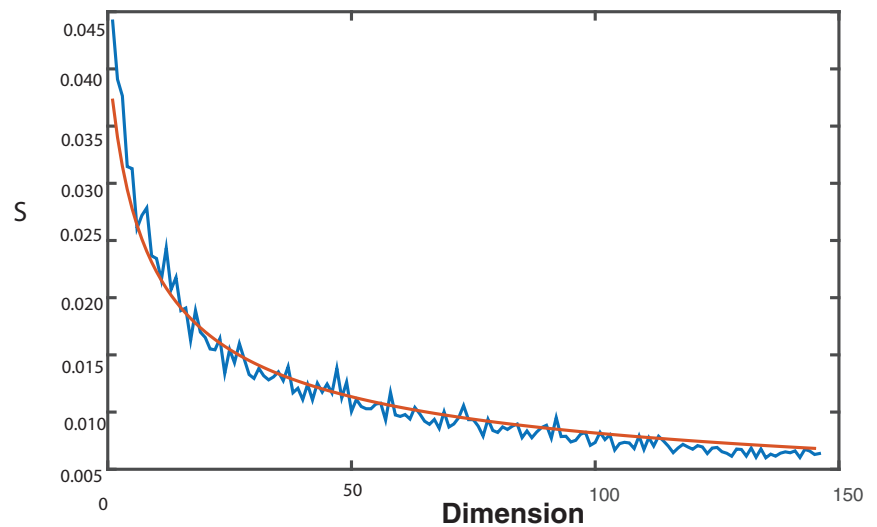

FIG. 9. (Color online) Fluctuations around the mean value of quantum discord in the fully chaotic phase space, $p=\pi / 2, \kappa=6$, as measured by the standard deviation as a function of the number of qubits $N$ (blue curve). The red line shows the $1 / \sqrt{N}$ behavior.

been shown to be instrumental in many quantum informationprocessing tasks [34].

\section{DISCUSSION AND SUMMARY}

The quantum kicked top is a simple but versatile system for studying various aspects of quantum chaos. A major advantage of this system is that it simulates a collection of $N=2 j$ qubits evolving in the symmetric subspace under an exchange of qubits. This allows for the possibility of studying different measures of quantum correlations such as entanglement and discord in the same system. The finite dimension of the Hilbert space makes it possible to perform accurate calculations without errors introduced due to truncations issues. In this paper we have shown that the dynamics of two-qubit discord in a multiqubit system collectively evolving as a quantum kicked top shows signatures of chaos. The discord between any two qubits shows quasiperiodic modulations for initial states localized in regular regions. The periodic oscillation is lost when the initial state lies in the chaotic sea, and the discord rapidly rises to an almost constant value. The time-averaged discord is higher when the initial conditions correspond to chaotic region of the classical phase space and the boundary between regular and choatic regions is sharply delineated by the change in the average discord.

Chaos occurs when the number of constraints or symmetries is fewer than the degrees of freedom. The same lack of symmetries at the quantum level means that the Hamiltonians cannot be described in block-diagonal form. Instead, chaotic Hamiltonians have eigenstatistics that are well described by random matrices [2,3]. Classical chaos can generate random probability distributions in phase space. Corresponding quantum dynamics can generate random states in Hilbert space [35]. When we focus our attention on the reduced subsystem of two qubits, this manifests as the generation of highly discordant states corresponding to the chaotic regions of the phase space. Quantum chaotic dynamics drives the system into arbitrary superposition of quantum states and this results in a higher average of value of discord in the chaotic part of the phase space as compared to the regular islands.

An interesting question that is relevant to quantum information-processing applications is the comparison of various measures of quantum correlations-the two most important ones being entanglement and discord. Our calculations show that the dynamical behavior of two-qubit discord mirrors very closely the two-qubit von Nuemann entropy but behaves opposite to the two-qubit concurrence. This raises interesting questions about the relationship of discord and concurrence, which we plan to explore in future work. Our results shed new light on the behavior of quantum correlations in chaotic systems, and since all parameters used are in an experimentally accessible regime, our work is relevant to future experiments exploring quantum chaos.

\section{ACKNOWLEDGMENTS}

We thank Dr. A. Hamel, Dr. Ravinder R. Puri, Dr. Marco Piani, Dr. Kevin Resch, Dr. Alexei Kaltchenko, and Dr. Animesh Datta for helpful discussions. This research was supported by the Natural Sciences and Engineering Research Council of Canada, the Ontario Ministry of Research and Innovation, and Wlifrid Laurier University. V.M. acknowledges the support of the Center for Quantum Information and Control (CQuIC) at the University of New Mexico.
[1] Edward Ott, Chaos in Dynamical Systems (Cambridge University Press, Cambridge, UK, 2002).

[2] F. Haake, Quantum Signatures of Chaos (Springer-Verlag, Berlin, 1991).

[3] O. Bohigas, M. J. Giannoni, and C. Schmit, Phys. Rev. Lett. 52, 1 (1984).

[4] T. Bhattacharya, S. Habib, and K. Jacobs, Phys. Rev. Lett. 85, 4852 (2000).

[5] W. H. Zurek and J. P. Paz, Phys. Rev. Lett. 72, 2508 (1994).

[6] A. Peres, Phys. Rev. A 30, 1610 (1984).

[7] R. Schack and C. M. Caves, Phys. Rev. E 53, 3257 (1996).

[8] C. Chamon, A. Hamma, and E. R. Mucciolo, Phys. Rev. Lett. 112, 240501 (2014).

[9] S. Ghose and B. C. Sanders, Phys. Rev. A 70, 062315 (2004).
[10] P. A. Miller and S. Sarkar, Phys. Rev. E 60, 1542 (1999).

[11] S. R. Hudson, Phys. Rev. E 76, 046211 (2007).

[12] X. Wang, S. Ghose, B. C. Sanders, and B. Hu, Phys. Rev. E 70, 016217 (2004).

[13] W. H. Zurek, Ann. Phys. (Berlin) 9, 855 (2000); H. Ollivier and W. H. Zurek, Phys. Rev. Lett. 88, 017901 (2001).

[14] Eli Biham et al., Theoretical Computer Science (Elsevier, Amsterdam, 2004).

[15] K. Modi, A. Brodutch, H. Cable, T. Paterek, and V. Vedral, Rev. Mod. Phys. 84, 1655 (2012).

[16] A. Datta, A. Shaji, and C. M. Caves, Phys. Rev. Lett. 100, 050502 (2008).

[17] S. Chaudhary et al., Nature 461, 768 (2009).

[18] Yu Chen et al., Phys. Rev. Lett. 113, 220502 (2014). 
[19] F. Haake, M. Kus, and R. Scharf, Z. Phys. B: Condens. Matter 65, 381 (1987)

[20] F. T. Arecci et al., Phys. Rev. A 6, 2211 (1972).

[21] Roy J. Glauber and Fritz Haake, Phys. Rev. A 13, 357 (1976).

[22] R. R. Puri, Mathematical Methods of Quantum Optics (Springer, Berlin, 2001).

[23] X. Wang and K. Molmer, Euro. Phys. J. D 18, 385 (2002).

[24] R. Horodecki, P. Horodecki, M. Horodecki, and K. Horodecki, Rev. Mod. Phys. 81, 865 (2007).

[25] William K. Wootters, Phys. Rev. Lett. 80, 2245 (1998).

[26] Stephen M. Barnett, Quantum Information (Oxford University Press, Oxford, 2009).
[27] A. Datta, Ph.D. thesis, University of New Mexico, 2008.

[28] V. Madhok and A. Datta, Phys. Rev. A 83, 032323 (2011).

[29] Y. S. Weinstein, S. Lloyd, and C. Tsallis, Phys. Rev. Lett. 89, 214101 (2002).

[30] M. Lombardi and A. Matzkin, Phys. Rev. E 83, 016207 (2011).

[31] M. Kus, J. Mostowski, and F. Haake, J. Phys. A 21, L1073 (1988).

[32] V. Madhok, C. A. Riofrío, S. Ghose, and I. H. Deutsch, Phys. Rev. Lett. 112, 014102 (2014).

[33] W. Wootters, Found. Phys. 20, 1365 (1990).

[34] J. Emerson et al., Science 302, 2098 (2003).

[35] A. Scott and C. Caves, J. Phys. A: Math. Gen. 36, 9553 (2003). 